\title{
Effect of Adding Nano Silver-Hydroxyapatite to the Orthodontic Primer on Bracket-Enamel Shear Bond Strength
}

\author{
Mahsa Asadi Haqhiqhat Gilani ${ }^{1}$, Nazila Ameli ${ }^{2}$, Raheb Ghorbani ${ }^{3}$, Azam Akhavan ${ }^{4}$, \\ Arman Rabiei ${ }^{5}$, Mehdi Salehi Zeinabadi ${ }^{6}$, Somayeh Kameli ${ }^{7}$
}

\begin{abstract}
${ }^{1}$ Student Research Committee, Dental School, Semnan University of Medical Sciences, Semnan, Iran. ${ }^{2}$ Department of Orthodontics, Dental School, Semnan University of Medical Sciences, Semnan, Iran. ${ }^{3}$ Social Determinants of Health Research Center, Semnan University of Medical Sciences, Semnan, Iran. ${ }^{4}$ Radiation Applications Research School, Nuclear Science and Technology Research Institute, Tehran, Iran. ${ }^{5}$ Department of Biomedical Engineering, Science and Research Branch, Islamic Azad University, Tehran, Iran. ${ }^{6}$ Department of Paediatric Dentistry, Dental School, Semnan University of Medical Sciences, Semnan, Iran. ${ }^{7}$ Department of Paediatric Dentistry, Dental School, Shahed University of Medical Sciences, Tehran, Iran.
\end{abstract}

\section{ABSTRACT}

\section{BACKGROUND}

Despite of advantages of orthodontic treatment and simplicity of the technique, there are still some defects. Disadvantages include aggregation of microbial plaques, white spot formation, and bond failure. Addition of decay decreasing particles to orthodontic bonding agent can be justified when the shear bond strength remains acceptable. We wanted to study the effect of adding different concentrations of nanosilver / hydroxyapatite to orthodontic primer on shear bond strength of bracketenamel and evaluate the location of bonding failure.

\section{METHODS}

Fourty sound premolar teeth were selected and assigned to four groups randomly. Group 1 : 3M - Z250 composite with Single Bond Universal Adhesive 3 M (control group); Group 2: bonding with $1 \%$ nano-silver / hydroxyapatite bonding; Group 3: bonding with $5 \%$ nano-silver / hydroxyapatite bonding; Group 4: bonding with 10 $\%$ nano-silver / hydroxyapatite. After bonding the brackets to the etched enamel surface, the shear bond strength was calculated using Instron Universal Testing Machine. The amount of remaining adhesive on the enamel surface was determined according to the Adhesive Remnant Index (ARI) scale. Data were analysed using the Shapiro-Wilk test, Kruskal-Wallis test, and associated multiple comparison test. SPSS 24 software was used and the significance level was set at 0.05 .

\section{RESULTS}

The shear bond strength showed significant differences in the four groups $(\mathrm{P}=$ 0.021). The strength of $1 \%$ concentration of nano-silver / hydroxyapatite $(\mathrm{P}=0.014)$ and concentration of $5 \%(\mathrm{P}=0.007)$ was lower than the control group. However, the concentration of $10 \%$ and the control group were not significantly different $(\mathrm{P}=$ 0.334). No significant difference observed in ARI in the four groups. $(P=0.859)$

\section{CONCLUSIONS}

Adding $10 \%$ nano-silver / hydroxyapatite to the orthodontic bonding primer would not affect bond strength adversely and can replace conventional bonding to reduce the risk of decay. In all groups, less than half of the adhesive remained on the enamel.

\section{KEY WORDS}

Nano-Silver / Hydroxyapatite, Shear Bond Strength, Orthodontic Resin Composite, Orthodontic Primer
Corresponding Author:

Dr. Nazila Ameli,

Associate Professor,

Department of Orthodontics,

Dental School, Semnan University

of Medical Sciences, Semnan, Iran.

E-mail: nazilaa.aameli@gmail.com

DOI: $10.14260 /$ jemds/2020/757

How to Cite This Article:

Gilani MAH, Ameli N, Ghorbani $R$, et al. Effect of adding nanosilver-hydroxyapatite to the orthodontic primer on bracketenamel shear bond strength. J Evolution Med Dent Sci 2020;9(46):3457-3462, DOI: 10.14260/jemds/2020/757

Submission 30-05-2020,

Peer Review 02-10-2020,

Acceptance 09-10-2020,

Published 16-11-2020.

Copyright (C) 2020 Mahsa Asadi Haqhiqhat Gilani et al. This is an open access article distributed under Creative Commons Attribution License [Attribution 4.0 International (CC BY 4.0)] 


\section{BACKGROUND}

Since the early days of orthodontics, the bonding technique of resin-based composites as the adhesive agent for the attachment of brackets to the teeth surfaces has been used in the orthodontic treatments. Moreover, composite resins are generally used by orthodontists because of their ease of use and reduced bonding time. Unfortunately, despite of advantages such as aesthetics and simplicity of the technique, there are still some defects. The disadvantages include aggregation of microbial plaques, white spot formation, and bond failure; these problems cause a prolongation of the treatment course, increased treatment costs, orthodontist and patient time-wasting, and decreased desirable aesthetic results. ${ }^{1-4}$

The debonding of bracket from the tooth enamel can occur either in the tooth-adhesive or the bracket adhesive junction. According to past studies, debonding at the bracket-adhesive junction is more prevalent. ${ }^{5-9}$

Nowadays, the application of nanotechnology systems in resin composites is one of the great advances in the field of dental materials. ${ }^{10,11}$ Several studies evaluated the effect of adding nanoparticles made of silver or titanium oxide into the composites in the aspects of antimicrobial features and bond strength; variable results were reported, from no change in the bond strength to a reduction or increase in that.1-5 Silver particles have been used in the treatment of microbial diseases. ${ }^{12,13}$ Along with demonstrated antimicrobial features of silver, its favourable physical effects on curing can improve the shear bond strength of the bracket enamel junction. Silver is a safe, antibacterial metal, because it is compatible with animal cells but very toxic to bacteria. Smaller nanoparticles of silver ( $<100$ nanometres) can establish a very close contact with microbes. Having higher surface-to-volume ratio than bigger particles, they provide a larger area of antimicrobial effect. ${ }^{14}$

Hydroxyapatite (HAP) is a crystalline calcium phosphate compound that forms the major part of the enamel mineral structure and higher than $60 \%$ of the dentin weight. The mineral matrix of human bones is also made of hydroxyapatite. When hydroxyapatite particles penetrate into the porosities of demineralized enamel they inhibit the release of calcium from the enamel surface, thereby increasing the tooth resistance to caries. Due to the features like similarity to the main mineral part of the human hard tissues, biocompatibility and low solubility in moist environments, hydroxyapatite is widely used in biological, medical, and dental applications. ${ }^{15}$

The antibacterial feature is an important characteristic of HAP, shown in different studies to restrict the growth of Streptococcus mutans and other oral cavity pathogens. ${ }^{16}$ The antimicrobial feature is very valuable particularly in orthodontic treatments, because the increased incidence of white spot lesions and caries around the dental brackets is a common problem in such treatments, ${ }^{9,17}$ so if a bonding material has antimicrobial and anti-cariogenic potentials, it will be noted. $18-20$

The shear bond strength is one of the best important characteristics of the materials used in bonding the orthodontic brackets. Only if hydroxyapatite does not affect the shear bond strength of the composite adversely, it is recommended to use it in orthodontic bracket bonding to reduce the caries incidence. ${ }^{11}$

In a study by Akhavan et al, they evaluated the effects of adding silver-hydroxyapatite nanoparticle to orthodontic adhesive on its shear bond strength. The results showed that 1 $\%$ concentration of silver-hydroxyapatite nanoparticle caused an elevation in the shear bond strength; while with increasing the concentration up to $10 \%$, undesirable effects were observed comparing to the control group. ${ }^{21}$ Another study by Blocher et al. compared the effect of application of silverhydroxyapatite nanoparticles in the orthodontic primers on the shear strength of the bracket bonding to enamel. The results showed no significant differences in the bond strength between the study groups and the control one.22

As silver-hydroxyapatite nanoparticles have the ability to inhibit the microbial growth, if they are able to have a positive effect on bracket bond strength, they can be absolutely appropriate alternatives for common composite bonds in orthodontics. In the present study, our purpose is to assess the shear bond strength of the enamel adhesive and bracket adhesive junctions by adding silver and hydroxyapatite nanoparticles in different concentrations, in order to decrease the incidence of dental caries developed following orthodontic treatments. Moreover, along with determining the shear bond strength of the bracket enamel junction, the fracture site can be important so, we used the Adhesive Remnant Index (ARI) score system to investigate the debonding format and compare the entire study groups to the control group according to the amount of the remained adhesive on the enamel surface after the debonding fracture test.

\section{METHODS}

In this in-vitro experimental study, 40 intact premolars extracted for orthodontic purposes in the age group of $10-30$ years, were collected (ethical code IR.SEMUMS.REC.1396.150) and were randomly divided into 4 groups (10 teeth in each group). In the control group (without silver-hydroxyapatite nanoparticles), after cleaning the buccal surfaces of the teeth and etching with $35 \%$ phosphoric acid (Ultradent-Ultra Etch, USA) for 30 seconds, teeth were rinsed for 10 seconds and dried using mild-pressure air. Then, the Single Bond Universal adhesive - $3 \mathrm{M}$ was applied on the surfaces using an applicator. The surfaces were cured for 10 seconds using the LED light curing device (L. E. Demetron, SDS Kerr, USA) at wavelength of $470 \mathrm{~nm}$ and light intensity of 1100 $\mathrm{mw} / \mathrm{cm}^{2}$. Finally, 3M - Z250 composite was placed on the base of the brackets, and the brackets were positioned on the centre of tooth using a bracket positioner (North American Braces, USA). After removing the excess composite with a probe, the adhesives on the bracket base were cured for 40 seconds (10 seconds for each side of the bracket).

In the group of $1 \%$ silver-hydroxyapatite concentration, the same steps were applied for preparing tooth surface. Then for the preparation of bonding with $1 \%$ concentration, the 10 $\%$ silver-hydroxyapatite nanoparticle powder (the average size of the silver and hydroxyapatite nanoparticles were 42 and $62 \mathrm{~mm}$, respectively) was weighted and mixed with the 
given amount of Single Bond Universal Adhesive - $3 \mathrm{M}$ bonding.

The resultant mixture was applied on the buccal surfaces using a micro-brush. After being cured using the light curing device, the brackets were bonded to the centre of the teeth with adhesive on their base. Following removal of the excess composite using a probe, the brackets were cured for 40 seconds (10 seconds for each side of the bracket).

In other groups, silver-hydroxyapatite nanoparticle powder was added in the concentrations of $5 \%$ and $10 \%$ of the total weight, respectively, and the brackets were bonded on the teeth similar to the group 2 . The samples were then mounted and incubated for 24 hours in distilled water at $37^{\circ} \mathrm{C}$.

A mounting jig was used to mount the teeth bonded to brackets in the same condition, and samples were mounted to $1 \mathrm{~mm}$ below Cemento Enamel Junction (CEJ), in a way that the bracket base areas were perpendicular to the horizon line.

To assess the shear strength, a chisel-like bar with a cutting edge of $0.5 \mathrm{~mm}$ thickness was connected to the head of Instron Universal Testing Machine (Zwick Roell, Germany) and a shear force of $0.5 \mathrm{~mm} /$ min speed was applied in the incisal-cervical orientation at the junction of bracket to tooth surface. The fracture force was calculated using Test Xpert V11.0 software in megapascals. After debonding, fracture surfaces were examined using an optical stereomicroscope under 10x magnification. Based on ARI score system (Artun and Bergland, Adhesive Remnant Index), the remnant adhesives were determined as the following:

0 - No adhesive left on the tooth.

1- Less than half of the adhesive left on the tooth.

2- More than half of the adhesive left on the tooth.

3- The entire adhesive is left on the tooth, and there is an obvious mark of the bracket mesh. ${ }^{23}$

\section{Statistical Analysis}

Analysis of data was performed using the Shapiro-Wilk test, Kruskal-Wallis test, and associated multiple comparison test. The software used was SPSS 24 and the significance level 0.05 .

\section{RESULTS}

Shear bond strength showed significant differences among the studied groups ( $\mathrm{P}=0.021)$ (Table 1$)$. The bond strengths of the composite with $1 \%$ silver-hydroxyapatite nanoparticle $(\mathrm{P}$ $=0.014)$ and also the $5 \%$ composite $(\mathrm{P}=0.007)$ were lower than the control group. While the shear bond strength of the composite with $1 \%$ nanoparticle demonstrated no significant difference with the control group $(\mathrm{P}=0.344)$ (Table 1$)$, also the strengths of composites with different concentrations of silver-hydroxyapatite nanoparticles had no significant difference.

The distribution of remnant amount of adhesive on the enamel after the bond fracture resistance test is shown in the Table 2. The distribution of adhesive remnant on the enamel after the debonding fracture test showed no significant difference among the four groups $(\mathrm{P}=0.859)$.

\begin{tabular}{|ccccccc|}
\hline $\begin{array}{c}\text { Concentration of } \\
\text { Nano-Silver / HA }\end{array}$ & $\mathbf{n}$ & Mean & SE & Median & IQR & $\begin{array}{c}\text { P } \\
\text { Value }\end{array}$ \\
\hline \% $\%$ & 10 & 27.8 & 5.4 & 27.6 & 30.9 & \\
$10 \%$ & 10 & 26.1 & 4.8 & 25.4 & 26.0 & 0.021 \\
Control & 10 & 43.2 & 6.3 & 34.9 & 35.3 & \\
\hline
\end{tabular}

Table 1. The Means, Standard Errors (SE), Medians and Interquartile Range (IQR) of Orthodontic Bracket to Enamel Shear Bond Strength in Different Concentrations of Silver-Hydroxyapatite Particles

\begin{tabular}{|ccccccccc|c|}
\hline $\begin{array}{c}\text { Remnant Adhesive } \\
\text { on Enamel }\end{array}$ & $\begin{array}{c}\text { Control } \\
\text { P }\end{array}$ & $\mathbf{1} \%$ & $\mathbf{1}$ & $\mathbf{5} \%$ & $\mathbf{1 0} \%$ \\
0 & - & - & - & - & - & - & 1 & 10 \\
1 & 2 & 20 & 1 & 10 & 1 & 10 & 1 & 10 \\
2 & 4 & 40 & 3 & 30 & 4 & 40 & 1 & 10 \\
3 & 4 & 40 & 6 & 60 & 5 & 50 & 7 & 70 \\
\hline $\begin{array}{c}\text { Table 2. Distribution of Adhesive Remnant on the Enamel after } \\
\text { Debonding Fracture Resistance Test in the Study Groups Based } \\
\text { on ARI Criteria }\end{array}$ \\
\hline
\end{tabular}

\section{DISCUSSION}

Despite extensive advances in orthodontics, the increased caries risk in the presence of orthodontic brackets is still an unsolved problem. The studies show that fixed orthodontic treatments result in increased colonization of Streptococcus mutans during the treatment course. ${ }^{24,25}$ The addition of anticariogenic nanoparticles to the bracket to enamel adhesives is an effective method of caries risk reduction in the fixed orthodontic treatment course, but it is only useful if the bracket to enamel shear bond strength does not decrease. ${ }^{5}$

The anti-cariogenic effects of silver-hydroxyapatite nano particles, $12,22,26,27$ are well demonstrated, so in the present study, we investigated the shear strength by adding nanoparticles in $1 \%, 5 \%$, and $10 \%$ concentrations to the orthodontic primers. The $10 \%$ concentration group was close to the control group in shear strength. The $1 \%$ and $5 \%$ concentration groups were also close in the shear strength but had significant differences with the control and $10 \%$ concentration groups. The debonding fracture sites were not different in the four groups. And more than half of the adhesives were left on the enamel surfaces in all of the groups after the debonding fracture test.

In a study by Sang lee et al, they investigated the antimicrobial effect of silver nanoparticles. ${ }^{13}$ They reported the positive effect of silver nanoparticles on Streptococcus mutans bacteria which are the initiators of dental caries. Moreover, in a study performed by Sharaf al-Din and Feizi to investigate the effect of adding hydroxyapatite nanoparticles on micro leakage of the class $\mathrm{V}$ restorations, they reported that the micro leakage amount was significantly decreased in the glass ionomer restorations modified with hydroxyapatite nanoparticles. ${ }^{28}$ The similar studies confirmed that silver and hydroxyapatite nanoparticles caused a caries risk reduction. ${ }^{12,13,22,26,27}$

In the study by Koohpeima et al, the effect of adding silver nanoparticles into dental composites on the shear strength was investigated using two different adhesive bonding methods. To achieve this, 90 premolars extracted for orthodontic purposes were collected. After the preparation of silver nanoparticles, the material internal structure was examined using Transmission Electron Microscope (TEM) and $\mathrm{x}$-ray, and then it was positioned directly on the exposed 
dentins. Next, the adhesive composites were used in two different ways, one system by etching and rinsing, and the other as a self-etch adhesive system. The shear strength test was calculated using Instron Universal Testing Machine. ${ }^{29}$ Subsequently, the addition of silver nanoparticles improved the shear strength in both study methods. In dentistry, both methods of material combination with nanoparticles and covering the material surface with nanoparticles were used to decrease the number of bacteria. ${ }^{30}$ Just the $1 \%$ concentration of silver nanoparticle was investigated in Koohpeima study, and it was reported to have positive effect on shear strength improvement. The appropriate shear strength was observed with the $10 \%$ nanoparticle concentration in our study.

In Kheur study, mechanical characteristics of the glass ionomer cement mixed with hydroxyapatite nanoparticles were evaluated. Based on previous studies, the elastic coefficient of the glass ionomer cement is lower than the resin composites, and in Kheur study, it was reported that the addition of hydroxyapatite nanoparticles improved the physical characteristics of the glass ionomer cement. They found that with hydroxyapatite nanoparticles added into the glass ionomer powder in $6 \%$ concentration, the shear bond strength of the glass ionomer was reported to be higher than the control group. ${ }^{31}$ In our study, the hydroxyapatite nanoparticle size was lower than the size range of nanoparticles in Kheur study. Moreover, the hydroxyapatite nanoparticles in our study were accompanied with silver nanoparticles.

Akhavan et al. studied the effect of adding silverhydroxyapatite nanoparticles on the shear bond strength of the orthodontic adhesives. They concluded that the application of silver-hydroxyapatite nanoparticles in the orthodontic adhesives in $1 \%$ and $5 \%$ concentrations resulted in increasing and maintaining of the shear bond strength, respectively. ${ }^{21}$ While increasing the concentration of nanoparticles to $10 \%$ had an adverse effect comparing to the control group. In Akhavan study, in which only the concentration of silver nanoparticles was changed, the shear strength of $1 \%$ concentration group was reported to be higher than the control group, and that of $5 \%$ group was close to the $1 \%$ group with just a little difference. In our study, the $10 \%$ silver-hydroxyapatite concentration was reported to be close to the control group. In Akhavan study, silver nanoparticles have a positive effect on the shear strength improvement, this can be explained that increasing the silver nanoparticle concentration can cause better curing and overcome the shadows of hydroxyapatite nanoparticles in the given concentration.

In the study by Golkar et al, they evaluated the effect of adding silver-hydroxyapatite nanoparticles on the mechanical characteristics of a kind of resin composite modified by glass (RMGI). The study results showed that the addition of $5 \%$ silver-hydroxyapatite nanoparticle improved the flexural strength, but no significant differences were observed in other concentrations $(1 \%, 2 \%, 5 \%, 7 \%$, and $10 \%)$. The preparation showed higher flexural and compressive strength after 1 week and 1 month. ${ }^{32}$ Favourable factors of the present study include using enough number of samples, different concentrations and the debonding fracture test. Due to release of fluoride, the use of glass ionomer base showed better results in caries prevention after orthodontic treatment but it showed lower strength than resin composites in the debonding fracture test. Ceramic brackets have a chemical bonding while stainless steel brackets have a mechanical bonding to the enamel surface, so addition of nanoparticles to the bracket adhesive can disrupt the chemical bonding and question the results.

Seyed Tabaii et al evaluated the effect of adding hydroxyapatite nanoparticles on the shear bond strength of the ceramic brackets bonded using Resin Modified Glass Ionomer (RMGI) cement. 33 The RMGI cement containing $10 \%$ NHA particle showed significantly lower shear bond strength than the other groups, however, the differences among other groups were not significant. Unlike the control group, the fracture patterns in RMGI groups were often cohesive. The study result was that the RMGI cement containing $5 \%$ hydroxyapatite nanoparticle can be utilized in the ceramic bracket bonding with comparable results with the resin composites. It was appropriate to use XT Trans bond as the control group in the study, while other concentrations of the nanoparticles could change the study results, also the friction coefficient of the ceramic and stainless steel brackets are different, so this factor can conflict the results of this study to ours.

Reddy et al studied the changes in shear bond strength of orthodontic adhesives by adding silver, zinc oxide and titanium oxide nanoparticles. ${ }^{34}$ The study results revealed significant differences between the control group and the studied groups. They concluded that the addition of different nanoparticles into the orthodontic adhesives in low concentrations can decrease the shear bond strength and cause a premature debonding of brackets from the tooth enamel. Three types of anti-cariogenic nanoparticles in same concentration were investigated in this study, while the other concentrations of this type of material could change the results.

Shin-Hye performed compared the antibacterial and physical characteristics of empirical primers containing chlorhexidine (CHX) or Ursolic Acid (UA) with commercial starters. The antimicrobial activities of three starters (TX,TX CHX and TX - UA) against Streptococcus mutans in planktonic phase and biofilm were investigated by determining the Minimal Inhibitory Concentrations (MICs) and carcinogenic bacteria and performing growth and biofilm experiments. To analyse the physical features, the shear bond strength and fracture shape were evaluated. ${ }^{35}$ TX - UA did not show a significant antimicrobial function comparing to TX, also no significant differences were observed in shear bond strengths and ARIs in the four groups. Another study by Tabaii, showed there was no significant difference in the ARI scores between the study groups, ${ }^{33}$ so with the correct debonding technique of the orthodontic brackets, we expect that most of the adhesive is left on the enamel surface after the bracket debonding.

Another study by Blocher et al. compared the effect of adding silver nano and micro particles into the orthodontic primers on the shear bond strength. ${ }^{22}$ They reported no significant differences between the study groups in the shear bond strength. Moreover, the debonding level of bracket to enamel was investigated in the same study based on ARI scoring system, but no significant differences were reported. The concentration of silver nanoparticle in the study was $1 \%$ and also using bovine incisors could have an impact on the study result. Bovine teeth are extremely similar to the human teeth but there are also considerable differences. The most 
common debonding fracture site from the enamel was the bracket adhesive junction, so that using ARI scoring system, in most of the specimens, more than $50 \%$ of the adhesive was left on the tooth surface after the debonding fracture test.

Presence of several differences between in vitro and in vivo conditions is a limitation of this study, so we cannot trust only on in vitro results; thus, it is suggested that in future studies, the characteristics of orthodontic adhesives containing silver-hydroxyapatite nanoparticles is investigated in vivo.

Also due to the limitations caused by bonding that should not be exposed to light, we could not investigate the homogeneity of the silver-hydroxyapatite nanoparticle bonding before applying it on the conditioned dental surfaces. Other concentrations of the silver-hydroxyapatite nanoparticles (higher than $10 \%$ ) can also be investigated regarding the results of the present study, because increasing in anti-cariogenic particle concentration can have a positive effect on caries risk reduction in orthodontic treatments.

\section{CONCLUSIONS}

The $10 \%$ silver-hydroxyapatite concentration group had a similar fracture resistance to the control group and can be used as a suitable alternative for the orthodontic bracket bonding, in order to decrease the caries risk in treatment courses. Less than half of the adhesives was left on the enamel surfaces in all of the four groups. In fact, the addition of silverhydroxyapatite nanoparticles had no effect on the bracket to enamel debonding fracture site.

Data sharing statement provided by the authors is available with the full text of this article at jemds.com.

This work was supported by the Deputy of Research and Sciences of the Semnan University of Medical Sciences by the grant number of 1305.

Disclosure forms provided by the authors are available with the full text of this article at jemds.com.

\section{REFERENCES}

[1] Zimmer BW, Rottwinkel Y. Assessing patient-specific decalcification risk in fixed orthodontic treatment and its impact on prophylactic procedures. Am J Orthod Dentofacial Orthop 2004;126(3):318-24.

[2] Demling A, Heuer W, Elter C, et al. Analysis of supra-and subgingival long-term biofilm formation on orthodontic bands. Eur J Orthod 2009;31(2):202-6.

[3] Allaker R. The use of nanoparticles to control oral biofilm formation. J Dent Res 2010;89(11):1175-86.

[4] Gama ACS, de Vito Moraes AG, Yamasaki LC, et al. Properties of composite materials used for bracket bonding. Braz Dent J 2013;24(3):279-83.

[5] Algera TJ, Kleverlaan CJ, Prahl-Andersen B, et al. The influence of different bracket base surfaces on tensile and shear bond strength. Eur J Orthod 2008;30(5):490-4.

[6] Ribeiro AA, Almeida LF, Martins LP, et al. Assessing adhesive remnant removal and enamel damage with ultraviolet light: an in-vitro study. Ame J Orthod Dentofacial Orthop 2017;151(2):292-6.
[7] Hofmann E, Elsner L, Hirschfelder U, et al. Effects of enamel sealing on shear bond strength and the adhesive remnant index: Study of three fluoride-releasing adhesives in combination with metal and ceramic brackets. J Orofac Orthop 2017;78(1):1-10.

[8] Dalaie K, Mirfasihi A, Eskandarion S, et al. Effect of bracket base design on shear bond strength to feldspathic porcelain. Eur J Dent 2016;10(3):351-5.

[9] Behnaz M, Dalaie K, Mirmohammadsadeghi H, et al. Shear bond strength and adhesive remnant index of orthodontic brackets bonded to enamel using adhesive systems mixed with TiO2 nanoparticles. Dental Press J Orthod 2018;23(4):43.e1-7.

[10] Padovani GC, Feitosa VP, Sauro S, et al. Advances in dental materials through nanotechnology: facts, perspectives and toxicological aspects. Trends Biotechnol 2015;33(11):621-36.

[11] Khurshid Z, Zafar M, Qasim S, et al. Advances in nanotechnology for restorative dentistry. Materials (Basel) 2015;8(2):717-31.

[12] Lee SM, Kim IR, Park BS, et al. Remineralization property of an orthodontic primer containing a bioactive glass with silver and zinc. Materials (Basel) 2017;10(11):1253.

[13] Lee SJ, Heo M, Lee D, et al. Preparation and characterization of antibacterial orthodontic resin containing silver nanoparticles. Applied Surface Science 2018;432:317-23.

[14] Kasraei S, Azarsina M. Addition of silver nanoparticles reduces the wettability of methacrylate and siloranebased composites. Braz Oral Res 2012;26(6):505-10.

[15] Kalita SJ, Bhardwaj A, Bhatt HA. Nanocrystalline calcium phosphate ceramics in biomedical engineering. Materials Science and Engineering: C 2007;27(3):441-9.

[16] Grenho L, Barros J, Ferreira C, et al. In vitro antimicrobial activity and biocompatibility of propolis containing nanohydroxyapatite. Biomed Mater 2015;10(2):025004.

[17] Kukleva MP, Shetkova DG, Beev VH. Comparative age study of the risk of demineralization during orthodontic treatment with brackets. Folia Med (Plovdiv) 2002;44(12):56-9.

[18] Kaviya S, Santhanalakshmi J, Viswanathan B, et al. Biosynthesis of silver nanoparticles using citrus sinensis peel extract and its antibacterial activity. Spectrochim Acta A Mol Biomol Spectrosc 2011;79(3):594-8.

[19] Rai M, Yadav A, Gade A. Silver nanoparticles as a new generation of antimicrobials. Biotechnol Adv 2009;27(1):76-83.

[20] Sharma VK, Yngard RA, Lin Y. Silver nanoparticles: green synthesis and their antimicrobial activities. Adv Colloid Interface Sci 2009;145(1-2):83-96.

[21] Akhavan A, Sodagar A, Mojtahedzadeh F, et al. Investigating the effect of incorporating nanosilver/nanohydroxyapatite particles on the shear bond strength of orthodontic adhesives. Acta Odontol Scand 2013;71(5):1038-42.

[22] Blöcher S, Frankenberger R, Hellak A, et al. Effect on enamel shear bond strength of adding microsilver and nanosilver particles to the primer of an orthodontic adhesive. BMC Oral Health 2015;15:42.

[23] Artun J, Bergland S. Clinical trials with crystal growth conditioning as an alternative to acid-etch enamel pretreatment. Am J Orthod 1984;85(4):333-40. 
[24] Spencer CG, Campbell PM, Buschang PH, et al. Antimicrobial effects of zinc oxide in an orthodontic bonding agent. Angle Orthod 2009;79(2):317-22.

[25] Bloom RH, Brown LR. A study of the effects of orthodontic appliances on the oral microbial flora. Oral Surg Oral Med Oral Pathol 1964;17:658-67.

[26] Sodagar A, Akhavan A, Hashemi E, et al. Evaluation of the antibacterial activity of a conventional orthodontic composite containing silver/hydroxyapatite nanoparticles. Prog Orthod 2016;17(1):40.

[27] Ahn SJ, Lee SJ, Kook JK, et al. Experimental antimicrobial orthodontic adhesives using nanofillers and silver nanoparticles. Dent Mater 2009;25(2):206-13.

[28] Sharafeddin F, Feizi N. Evaluation of the effect of adding micro-hydroxyapatite and nano-hydroxyapatite on the microleakage of conventional and resin-modified Glassionomer $\mathrm{Cl} \mathrm{V}$ restorations. J Clin Exp Dent 2017;9(2):e242-8.

[29] Fatemeh K, Javad MM, Samaneh K. The effect of silver nanoparticles on composite shear bond strength to dentin with different adhesion protocols. J Appl Oral Sci 2017;25(4):367-73.

[30] Hamouda IM. Current perspectives of nanoparticles in medical and dental biomaterials. J Biomed Res 2012;26(3):143-51.
[31] Kheur M, Kantharia N, Lakha T, et al. Evaluation of mechanical and adhesion properties of glass ionomer cement incorporating nano-sized hydroxyapatite particles. Odontology 2019;108(1):66-73.

[32] Basir MM, Ataei M, Rezvani MB, et al. Effect of incorporation of various amounts of nano-sized hydroxyapatite on the mechanical properties of a resin modified glass ionomer. Shahid Beheshti Uni Dent J 2013;30(4):216-23.

[33] Tabaii ES, Sari MN. Evaluation of shear bond strength of resin reinforced glass ionomer cement modified by nanohydroxyapatite on ceramic bracket debonding using fulldimension wire. Annual Research \& Review in Biology 2014;4(10):1578-86.

[34] Reddy AK, Kambalyal PB, Patil SR, et al. Comparative evaluation and influence on shear bond strength of incorporating silver, zinc oxide, and titanium dioxide nanoparticles in orthodontic adhesive. J Orthod Sci 2016;5(4):127-31.

[35] Chung SH, Cho S, Kim K, et al. Antimicrobial and physical characteristics of orthodontic primers containing antimicrobial agents. Angle Orthod 2017;87(2):307-12. 